\title{
Bayesian inversion of synthetic AVO data to assess fluid and shale content in sand-shale media
}

\author{
Hafiz Mubbasher Anwer ${ }^{1}$, Aamir Ali ${ }^{1, *}$ and Tiago M Alves ${ }^{2}$ \\ ${ }^{1}$ Department of Earth Sciences, Quaid-i-Azam University, Islamabad 45320, Pakistan. \\ ${ }^{2} 3 D$ Seismic Lab, School of Earth and Ocean Sciences, Cardiff University, Cardiff, CF10 3AT, UK. \\ ${ }^{*}$ Corresponding author. e-mail: aamirali.geo@gmail.com
}

Reservoir characterization of sand-shale sequences has always challenged geoscientists due to the presence of anisotropy in the form of shale lenses or shale layers. Water saturation and volume of shale are among the fundamental reservoir properties of interest for sand-shale intervals, and relate to the amount of fluid content and accumulating potentials of such media. This paper suggests an integrated workflow using synthetic data for the characterization of shaley-sand media based on anisotropic rock physics (T-matrix approximation) and seismic reflectivity modelling. A Bayesian inversion scheme for estimating reservoir parameters from amplitude vs. offset (AVO) data was used to obtain the information about uncertainties as well as their most likely values. The results from our workflow give reliable estimates of water saturation from AVO data at small uncertainties, provided background sand porosity values and isotropic overburden properties are known. For volume of shale, the proposed workflow provides reasonable estimates even when larger uncertainties are present in AVO data.

\section{Introduction}

Sand-shale lithological sequences are a prospective hydrocarbon bearing play type in petroleum exploration (Quirein et al. 2012; Bjørlykke 2015; Diasty et al. 2015; Javanshir et al. 2015; Asim et al. 2016). Reservoir characterization of such lithological sequences has always challenged the geoscientists due to the presence of anisotropy and heterogeneity (Alves et al. 2014; Auchter et al. 2016; Kneller et al. 2016). The accurate reservoir performance prediction requires the use of reliable reservoir models by incorporating all the available data (Lim 2005). Knowing this, the potential of exploiting petroleum resources of a sand-shale sequence is critically dependent upon the accurate estimation of medium parameters, i.e., volume of shale ( $\left.V_{\text {shale }}\right)$ and water saturation $\left(S_{w}\right)$. The productivity of a sand-shale reservoir is strongly dependent on the distribution and amount of the shale and water within the sand (Sams and Andrea 2001).

There have been numerous attempts to estimate these important reservoir properties from seismic, well-log and core data (Patnode and Wyllie 1950; Winsauer and McCardell 1953; Wyllie and Southwick 1954; Waxman and Smits 1968; Poupon and Leveaux 1971; Van Golf-Racht 1982; Clavier et al. 1984; Worthington 1985; Herron 1986; Sen et al. 1988; Schwartz and Sen 1988; Bachrach 2006; Sengupta and Bachrach 2007; Alimoradi et al. 2011; Dandekar 2013). These attempts focused on developing a model based on the distribution of shale in pore space of the sandstone (Soto et al. 2010). However, as shales are very

Keywords. Rüger's approximation; shaley-sand VTI medium; seismic anisotropy; seismic Bayesian inversion; seismic AVO data. 


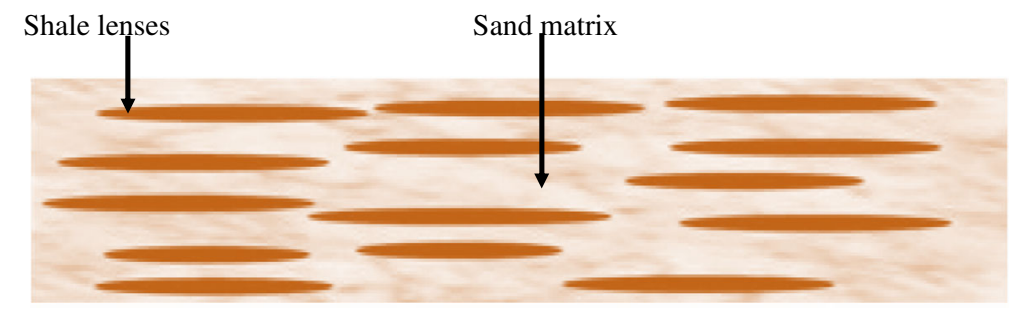

Figure 1. A conceptual model describing the shaley-sand medium with shale lenses aligned horizontally making a VTI medium.

heterogeneous and strongly anisotropic, core and well data alone may not be sufficient to map the subsurface shale sequences (Kumar and Hoversten 2012). Remote seismic measurements may play a central role in helping to characterize sand-shale media, but this will require a good understanding of relevant rock physics and scaling issues (Ali et al. 2011; Ali and Jakobsen 2011a, b; Kumar and Hoversten 2012). For instance, Takahashi (2000) have predicted sand-shale ratio based on statistical rock physics simulations of various bedding scenarios. Vernik et al. (2002) predicted net-to-gross values from $\mathrm{P}$ and $\mathrm{S}$ impedance inversion results. Stovas et al. (2004) used effective medium theory combined with Gassmann theory to predict netto-gross and saturation from amplitude vs. offset (AVO) attributes.

Rock-physics models are often used to link seismic data to reservoir properties (Avseth et al. 2005). However, this requires statistical techniques because simple rock-physics models using deterministic approach cannot capture the complexity of the sediment (Bachrach 2006). It is worth mentioning that seismic-based estimates of reservoir properties can be quite uncertain, but inclusion of rock physics modelling reduces the level of uncertainty by reducing the number of unknown parameters. In other words, rock physics modelling represents a kind of regularization within the context of seismic inversion (Ali and Jakobsen 2011a, b).

The objective of this paper is to propose a methodology for estimating the parameters $\left(V_{\text {shale }}\right.$ and $\left.S_{w}\right)$ of shaley-sand media using anisotropic rock physics modelling and synthetic AVO data. Our further aim is to investigate the level of uncertainty for anisotropic AVO data to recover these parameters. For this purpose, we have assumed a reservoir model with shale lenses embedded in sand background making a vertically transversely isotropic (VTI) medium as shown in figure 1.

Traditionally, sand-shale sequences are modelled in the form of alternating layers (Luo et al. 2005; Wang et al. 2006; Wang 2011), but our model

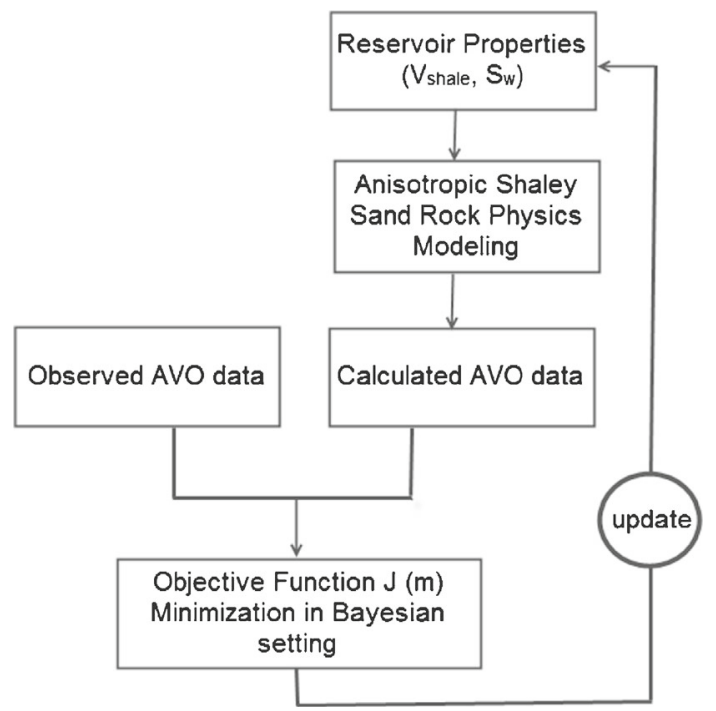

Figure 2. Schematic workflow diagram for estimation of reservoir (shaley-sand) parameters from AVO data.

takes in to account the effect of shale in the form of lenses. The layout of reservoir architecture for which we have developed the rock physics model is similar to a model described by Sayers (1998), in which lenses are laterally/vertically continuous over lengths smaller as compared to the seismic wavelengths. Such types of models are very important for sandstone reservoirs, where shale inclusions are present in the form of lenses (Ikelle et al. 1993) and vary from millimetres to tens of meters in thickness and from millimetres to kilometres in lateral extent (Potter et al. 2012). A field example of this assumed sand-shale model was referred by Haldorsen et al. (1987). Assakao sandstone, which contains a large number of near horizontal non-continuous shale lenses. The depositional environment was reported to be fluvial, mainly braided streams, but also meandering (Haldorsen et al. 1987).

The workflow followed to meet the objectives is given in figure 2. We have used anisotropic Tmatrix approximation for composite porous media 
(shaley-sand) along with relations of Wood (1955) and Brown and Korringa (1975) to obtain effective elastic properties (Jakobsen et al. 2003a, b; Ali and Jakobsen 2011a, b). Shale lenses are modelled in the form of ellipsoidal inclusions having large aspect ratios. Rüger's approximation of reflection coefficients for VTI media was used to obtain AVO data, i.e., reflection coefficients from top of the reservoir (Rüger 1998; 2002). A Bayesian inversion scheme for estimating reservoir parameters $\left(V_{\text {shale }}\right.$ and $\left.S_{w}\right)$ from synthetic AVO data was used to obtain the information about uncertainties as well as the most likely values (Aster et al. 2005; Tarantola 2005).

The outline of this paper is as follows. In section 2, we discuss how to estimate the effective elastic properties of shaley-sand media. Thereafter, we explained how we can use these elastic properties to generate synthetic AVO data. In section 3, we provide information on the Bayesian inversion method for estimation of reservoir parameters of shaley-sand media. We then present numerical results and discussion in section 4 . At the end of the paper, we draw conclusions based on the outcomes of forward and inverse modelling in the context of reservoir characterization for shaley-sand media.

\section{Forward problem}

The nonlinear forward problem is defined by

$$
\mathbf{d}=\mathrm{G}(\mathbf{m}) \text {. }
$$

Here, $\mathbf{d}$ is a vector of observable quantities (AVO data) and $\mathbf{m}$ is a vector of model parameters related to $V_{\text {shale }}$ and $S_{w}$ over the model space $\mathbf{M}$, i.e., $\mathbf{m} \in \mathbf{M}$. The operator $\mathrm{G}$ is a combination of rock physics modelling and seismic attribute generation. In the following subsections, we discuss the rock physics modelling for the shaley-sand media.

\subsection{Effective elastic properties of shaley-sand formation}

The rock physics modelling process provides a realistic and systematic basis for seismic attribute generation and interpretation (Avseth et al. 2005). Rock physics models are a key in linking the changes in lithology, porosity, permeability and pore fluid to changes in the velocities and density (Uden et al. 2004). Our shaley-sand reservoir model consists of a porous matrix with a population of high aspect ratio shale lenses with sand in the background. Elastic properties of sand matrix have

Table 1. Showing elastic properties of solid mineral, dry porous matrix, fluid and isotropic overburden used for calculation of reflection coefficients.

\begin{tabular}{lccccc}
\hline Material & $\begin{array}{c}\text { Density } \\
\left(\mathrm{kg} / \mathrm{m}^{3}\right)\end{array}$ & $\begin{array}{c}\text { Shear modulus } \\
(\mathrm{GPa})\end{array}$ & $\begin{array}{c}\text { Bulk modulus } \\
(\mathrm{GPa})\end{array}$ & $\begin{array}{c}\mathrm{C}_{11} \\
(\mathrm{GPa})\end{array}$ & $\begin{array}{c}\mathrm{C}_{44} \\
(\mathrm{GPa})\end{array}$ \\
\hline Solid mineral (quartz) & 2650 & 44 & 37 & 95.7 & 44 \\
Dry porous matrix (sand) & 2490 & 18 & 19.7 & 43.7 & 18 \\
Fluid (water/brine) & 1000 & 0.0 & 2.2 & 2.2 & 0.0 \\
Isotropic shale (overburden) & 2350 & 8.0 & 13.3 & 24 & 8.0 \\
\hline
\end{tabular}
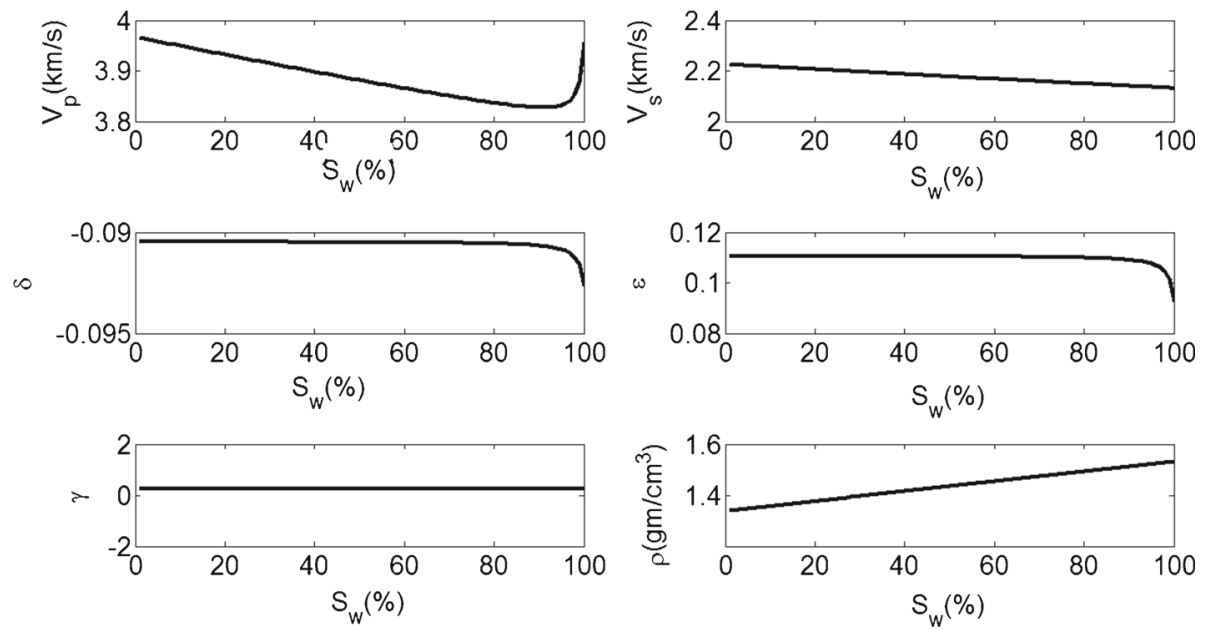

Figure 3. Variation of vertical velocities, Thomsen's anisotropic parameters and effective density as a function of $S_{w}$ for $20 \%$

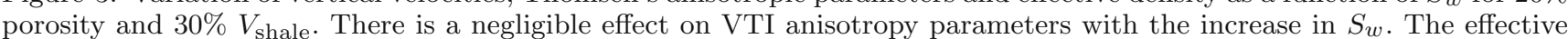
density increases with increasing water saturation as pores are replaced with heavier fluid (water). 
been obtained from Rüger (2002) for the dry case, which are listed in table 1 along with the properties of solid mineral, fluid and overburden. The included shale lenses having aspect ratio of $1 / 10$ within the sand matrix show horizontally aligned orientation, thus making a transversely isotropic (TI) medium with a vertical axis of symmetry characterized by five independent elastic constants. Moreover, the rationale behind known aspect ratio of shale lenses stems out from the fact that such information can potentially be obtained from geological field and core studies. The effective properties of a porous medium with inclusions (shale lenses) for the dry case are calculated using the T-matrix approach developed by Jakobsen et al. (2003a) given in Appendix A. The effects of fluid saturation on effective properties are modelled with the help of anisotropic relations of Brown and Korringa (1975) given in Appendix B.

A good understanding of the relevant rock physics modelling plays a central role in the characterization of sand-shale reservoirs and it should give us realistic values of effective stiffness. The tensors of the effective elastic stiffness constants can be viewed as a function of model parameters $\left(V_{\text {shale }}, S_{w}\right)$ related to shaley-sand media. The five independent elastic constants of a VTI medium can be expressed in two notations, either in terms of stiffness constants $\left(\mathrm{C}_{11}, \mathrm{C}_{13}, \mathrm{C}_{33}, \mathrm{C}_{55}\right.$ and $\left.\mathrm{C}_{66}\right)$ or of two vertical velocities $\left(V_{p}, V_{s}\right)$ and three Thomsen anisotropic parameters $(\gamma, \delta, \varepsilon)$ (Tsvankin 1997a, b). Here, we have followed the second notation and plotted these parameters and effective density $(\rho)$ of composite porous medium as a function of $S_{w}$ and $V_{\text {shale }}$ (figures 3-4). Thomsen's VTI anisotropic parameters $(\varepsilon, \gamma)$ representing $\mathrm{P}$ - and
S-wave anisotropies increase with increasing anisotropy (shale lenses); whereas the anisotropic parameter $\delta$, which controls the near-angle wave propagation decreases with increasing anisotropy (shale lenses). On the other hand, increasing water saturation has negligible impact on Thomsen's anisotropic parameters (figures 3-4). The variation of Thomsen's anisotropic parameters with saturation depends largely on the storage porosity of the background rock (Thomsen 1995; Cardona 2002). Smaller storage porosity in the background rock will result in a larger fluid pressure, and thus a reduction in the anisotropy coefficients (Thomsen 1995; Cardona 2002).

In this study, we have assumed fixed storage porosity of the background sand and homogenous mixing of fluids (water + gas). We also observed that the estimated $\mathrm{P}$-wave and $\mathrm{S}$-wave velocities decrease with the increasing volume of shale and water saturation. Moreover, the effective density $(\rho)$ of the shaley-sand medium increases with increasing water saturation, as gas having low density is being replaced by heavier fluid (water; figure 3 ). Figure 4 also reveals that as the shale content increases, the effective density decreases due to low density of shales as compared to sands. These trends are more or less as expected, but there are some nonlinear effects that would have been difficult to quantify without a suitable rock physics model for the composite porous medium.

\subsection{Seismic response of shaley-sand formations}

Surface seismic measurements play an important role in mapping the subsurface, tracing shaley-sand
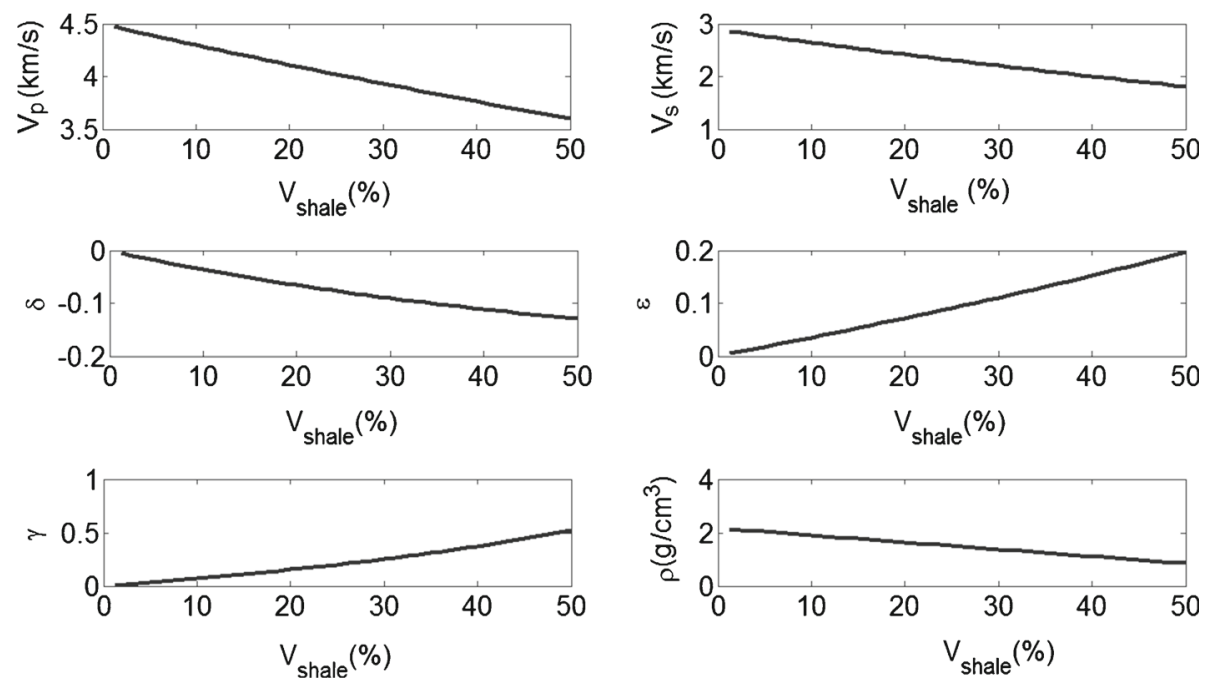

Figure 4. Variation of vertical velocities, Thomsen's anisotropic parameters and effective density as a function of $V_{\text {shale }}$ for $20 \%$ porosity and $20 \% S_{w}$. P- and S-wave anisotropies $(\varepsilon$ and $\gamma$ ) increase with increasing anisotropy due to shale lenses. The vertical velocities decrease with increasing volume of shale. Effective density decreases with increasing $V_{\text {shale, }}$ as sand is being replaced by shale. 
facies changes and visualizing reservoirs. AVO modelling has a great potential to provide important information within the context of reservoir characterization (Besheli et al. 2005). It is the most widely used technique for hydrocarbon detection, lithology identification and fluid parameter analysis (Feng and Bancroft 2006) Variations in the elastic properties of rocks caused by the shaleysand medium can be modelled using synthetic AVO data. Such a modelling technique will require sophisticated processing steps to be performed on seismic data due to the presence of multiples interfering constructively and destructively with primary reflections in many cases. In this study, we have used reflection coefficient as a seismic attribute to detect variations in the elastic properties of shaley-sand media. The shale lenses in our model are aligned horizontally in sand matrix to make it a medium of VTI symmetry. To calculate the reflection coefficients as a function of incidence angle $(i)$ for a plane wave scattered by a medium with vertical symmetry axis, a linearized

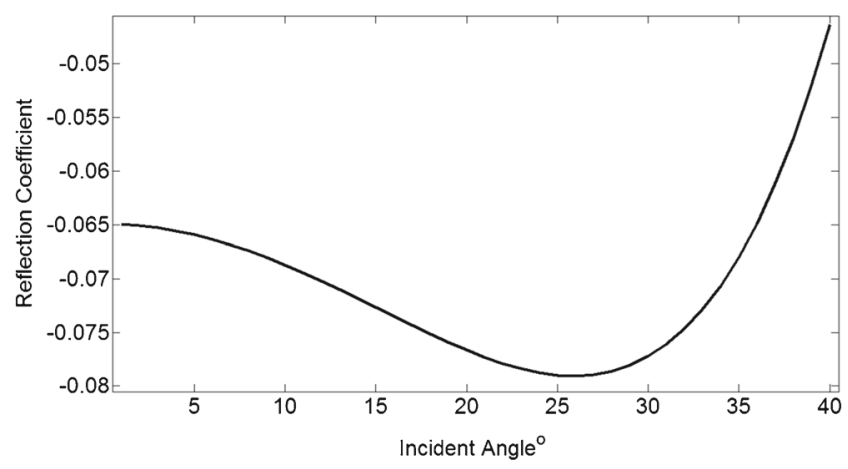

Figure 5. PP reflection coefficients vs. angle of incidence (AVO data) for $20 \%$ porosity, $20 \% S_{w}$ and $30 \% V_{\text {shale. }}$ The overburden properties used for the computation of PP reflection coefficients are given in table 1 . approximation is given by Rüger $(1998,2002)$ in the form:

$$
\begin{aligned}
R_{\mathrm{P}}^{\mathrm{VTI}}(i)= & \frac{1}{2} \frac{\Delta Z}{\bar{Z}}+\frac{1}{2}\left\{\frac{\Delta V_{\mathrm{P} 0}}{\bar{V}_{\mathrm{P} 0}}-\left(\frac{2 \bar{V}_{\mathrm{S} 0}}{\bar{V}_{\mathrm{P} 0}}\right)^{2} \frac{\Delta G}{\bar{G}}+\Delta \delta\right\} \\
& \times \sin ^{2} i+\frac{1}{2}\left\{\frac{\Delta V_{\mathrm{P} 0}}{\bar{V}_{\mathrm{P} 0}}+\Delta \varepsilon\right\} \sin ^{2} i \tan ^{2} i .(2)
\end{aligned}
$$

Here, $Z$ is the P-wave impedance, $G$ is the shear modulus, $V_{P 0}$ and $V_{S 0}$ are the vertical $\mathrm{P}$ - and $\mathrm{S}$ wave velocities. The constants $\varepsilon$ and $\delta$ are the anisotropy parameters for VTI media. The symbol $\Delta$ denotes contrast across an interface $\left(\Delta Z=Z_{2}-\right.$ $Z_{1}$ ) and a bar over a symbol $Z$ denotes the average $\left(\bar{Z}=\frac{Z_{1}+Z_{2}}{2}\right)$, where subscript 1 corresponds to the upper-half space and subscript 2 corresponds to lower-half space. Equation (2) can be used if the anisotropy and the velocity across the reflector are weak. An example of reflection coefficient data obtained using equation (2) is shown in figure 5 . There is a rapid decreasing trend for increasing incident angle till $30^{\circ}$ for the reflection coefficient curve before it begins to increase for higher angle of incidence.

\section{The inverse problem}

The inverse problem consists of estimating the model parameters $\mathbf{m}$ related to shaley-sand media $\left(V_{\text {shale, }} S_{w}\right)$ from the AVO data $\mathbf{d}$, such that $\mathrm{G}(\mathbf{m})=\mathbf{d}$. The solution to the inverse problem in a Bayesian setting is given by the posterior probability distribution $q(\mathbf{m} \mid \mathbf{d})$ over the model space $\mathbf{M}$. $q(\mathbf{m} \mid \mathbf{d})$ basically carries all the information about the model originating from two sources. The first source is the data $\mathbf{d}$ and information is given by the likelihood function. The second source is the prior information, which is expressed through a

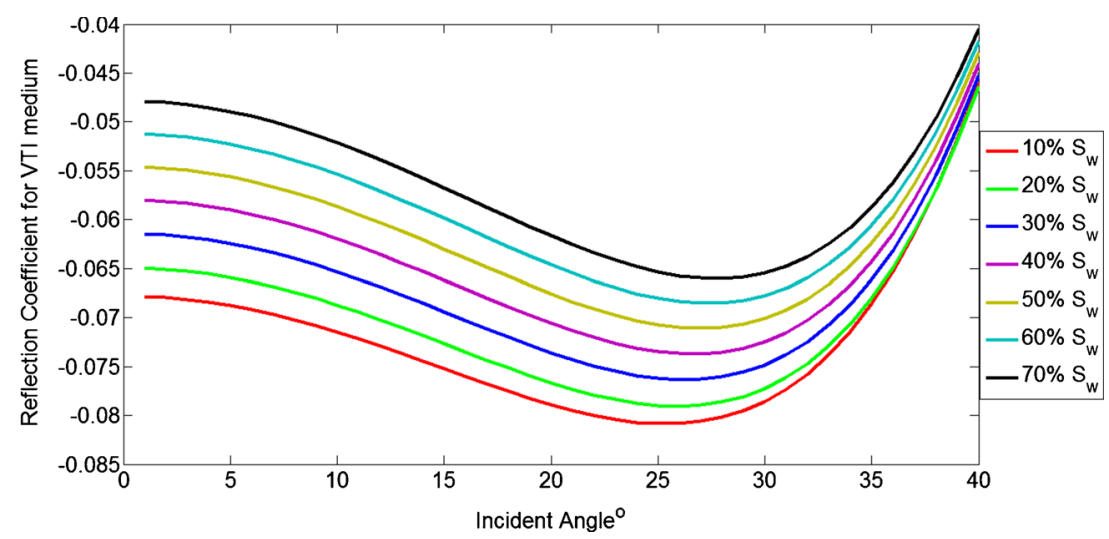

Figure 6. Variation of reflection coefficients as a function of angle for different percentages of $S_{w}$ with $20 \%$ porosity, $30 \%$ $V_{\text {shale }}$ and overburden properties as given in table 1. 
probability density function. More specifically, the posterior probability density function (PDF) represents the degree of belief about the possible values of $\mathbf{m}$ before and after observing the data $\mathbf{d}$ defined as (Aster et al. 2005):

$$
q(\mathbf{m} \mid \mathbf{d})=N \cdot \mathrm{e}^{-J(\mathbf{m})},
$$

where $N$ is a normalization constant and the objective function $J(\mathbf{m})$ in the case of Gaussian statistics is given by Aster et al. (2005) such as:

$$
\begin{aligned}
J(\mathbf{m})= & \frac{1}{2}\left[(\mathrm{G}(\mathbf{m})-\mathbf{d})^{\mathrm{T}} \mathrm{C}_{\mathrm{D}}^{-1}(\mathrm{G}(\mathbf{m})-\mathbf{d})\right. \\
& \left.+\left(\mathbf{m}-\mathbf{m}_{\mathbf{o}}\right)^{\mathrm{T}} C_{M}^{-1}\left(\mathbf{m}-\mathbf{m}_{\mathbf{o}}\right)\right] .
\end{aligned}
$$

Here, $\mathbf{m}_{\mathbf{o}}$ is defined as the mean value of the a priori distribution and $C_{D}$ and $C_{M}$ are the covariance matrices for the data and the model parameters, respectively. For the case of a non-informative prior, equation (4) can be changed as follows:

$$
J(\mathbf{m})=\frac{1}{2}\left[(\mathrm{G}(\mathbf{m})-\mathbf{d})^{\mathrm{T}} \mathrm{C}_{\mathrm{D}}^{-1}(\mathrm{G}(\mathbf{m})-\mathbf{d})\right] .
$$

In this study, we have considered a noninformative prior distribution. The rationale behind assuming a non-informative prior is that we do not intend to constrain our inversion scheme by incorporating prior information (obtained mostly from $\log$ /core data) about $V_{\text {shale }}$ and $S_{w}$. Therefore, in this scenario under the principle of indifference, all model parameter values have equal likelihood and covariance matrix $C_{M}$ for the model space will not be used in formulation of inverse problem. Also, we have used the covariance matrix $C_{D}$ for the data in the form when noise in the measured (seismic) data is independent and normally distributed with a standard deviation $\sigma$. So, with non-informative prior and normally distributed measurement errors (with standard deviation $\sigma$ ), the form of the objective function $J(\mathbf{m})$ to be minimized is given by the relation (Aster et al. 2005):

$$
J(\mathbf{m})=\min \sum_{i=1}^{n} \frac{\left((\mathrm{G}(\mathbf{m}))_{i}-\mathbf{d}_{i}\right)^{2}}{2 \sigma^{2}} .
$$

The normalization constant $N$ which appeared in equation (3) can be found by integration. Assume that the model parameters $\mathbf{m}$ are defined in the model space $\mathbf{M}$, i.e., $\mathbf{m} \in \mathbf{M}$, then the posterior distribution will satisfy (Aster et al. 2005):

$$
\int_{\mathbf{M}} \mathbf{q}(\mathbf{m} \mid \mathbf{d}) \mathbf{d} \mathbf{m}=\mathbf{1} \Rightarrow N=\frac{\mathbf{1}}{\int_{\mathbf{M}} \exp (-J(\mathbf{m}) \mathbf{d m})} \text {. }
$$

The inverse problem using the AVO data is ill-conditioned and sometimes ill-posed, raising questions about the reliability of the estimates (Downton et al. 2007). Therefore, it is very important to perform the uncertainty analysis of the predicted estimates. To quantify the prediction uncertainty in the estimates of model parameters, an assessment of the full posterior distribution $q(\mathbf{m} \mid \mathbf{d})$ is required. Since the number of parameters to be inverted in this paper are small, we have used numerical integration method (Ali et al. 2011, 2015) for full exploration of posterior distribution $q(\mathbf{m} \mid \mathbf{d})$, which yields the marginal PDFs for small dimensional problems (Tarantola 2005). Subsequently, we present an adaptation of the numerical integration method to the particular inverse problem used in this study (inverting for $V_{\text {shale }}$ and $S_{w}$ ). The marginal PDFs for the volume of shale and water saturation can be obtained in the following way. Let $V_{s h}$ denote the volume of shale, $S_{w}$ denote the water saturation and $\mathbf{M}$ is the model space for the parameters, the posterior distribution satisfies equation (7) written as:

$$
\int_{0}^{1} \int_{0}^{1} q\left(V_{s h}, S_{w}\right) d \_v_{s h} d \_s_{w}=1 .
$$

The marginal distribution for the volume of shale will then be obtained by integrating over all the water saturation written as:

$$
q\left(V_{s h}\right)=\int_{0}^{1} q\left(V_{s h}, S_{w}\right) d \_s_{w} .
$$

Similarly, the marginal distribution for water saturation can be obtained by integrating over all the volume of shale, such as:

$$
q\left(S_{w}\right)=\int_{0}^{1} q\left(V_{s h}, S_{w}\right) d_{-} v_{s h} .
$$

Marginal PDFs obtained via numerical integration require dense discretization of the model space. Sparse discretization of model space may lead to missing of important features of posterior distribution. This method is only useful when the computation of forward model is not much time consuming and we have a small dimensional problem at our hand. For high dimensional problems and time consuming forward problem, this method might prove to be ineffective and one may use Monte Carlo Markov chain methods (Ali et al. 2015).

\section{Numerical results and discussion}

We assumed a shaley-sand model with horizontally aligned shale lenses (inclusions) with the main aim to estimate the reservoir parameters $\left(V_{\text {shale }}\right.$ and $S_{w}$ ) and their associated uncertainty from AVO data. The elastic properties of dry porous matrix (sand) and overburden (shale) properties used in this study are given in table 1 . The micro porosity of shaley-sand model was assumed to be $20 \%$. The aspect ratio of shale lenses was set to be $1 / 10$. 
Effective elastic properties of shaley-sand were obtained by using the methodology discussed in subsection 2.1. In our T-matrix rock physics modelling, we have assumed an isotropic correlation function for modelling shale lenses. This assumption has nothing to do with the initial assumption of strongly elliptic shale inclusion resulting in a VTI medium. The correlation function only gives the probability of finding the elliptic shale inclusion or spatial distribution of elliptic shale inclusion. It can have ellipsoidal or spherical symmetry represented by the choice of aspect ratios. An isotropic (aspect ratio $=1$ ) correlation function means that there is an equal probability of finding the elliptic shale inclusion in all directions. We can also take an anisotropic correlation function, which will mean that possibility of finding the elliptic shale inclusion will change with the direction.

The sensitivity of effective elastic properties $\left(V_{p}, V_{s}, \varepsilon, \delta\right.$ and $\left.\gamma\right)$ and composite density $(\rho)$ of shaley-sand medium as a function of reservoir parameters $\left(V_{\text {shale }}\right.$ and $\left.S_{w}\right)$ to be estimated are presented in figures $3-4$. Using the effective elastic properties obtained via rock physics modelling, AVO data in the form of reflection coefficients as a function of incidence angle was generated from Rüger's approximation as described in subsection 2.2. We assumed an overburden composed of isotropic shale with the properties listed in table 1. In reality, shales are often found to behave elastically as transversely isotropic media with a vertical axis of symmetry (Jakobsen and Johansen 1999, 2000), but this simplifying assumption of an isotropic overburden does not affect the anisotropy of the shaley-sand reservoir. However, this assumption can affect the synthetic AVO data in terms of changing values of reflection coefficients.

For the sensitivity of AVO data (reflection coefficients) with the reservoir parameters $\left(V_{\text {shale, }}\right.$ $S_{w}$ ), different reflection coefficient curves were generated for varying percentages of one parameter keeping the other fixed. Figure 6 shows the variation of reflection coefficient for a range of $S_{w}$ starting from 10-70\% with fixed percentage of $V_{\text {shale }}$ equal to $30 \%$. Similarly, figure 7 shows the variation of reflection coefficient for a range of $V_{\text {shale }}$ starting from 10 to $90 \%$ with fixed percentage of $S_{w}$ equal to $20 \%$. These different reflection (AVA) curves help to explain two main things; intercept (normal incidence $\mathrm{P}$-wave reflectivity) and slope of curve (gradient) explaining how amplitude of reflection changes with angle. The gradient of each reflection curve is almost same for different percentages of $S_{w}$ showing less sensitivity. Reflection curves for different percentages of $V_{\text {shale }}$ behave differently in a sense that most curves show increasing trend with increase of $V_{\text {shale }}$. The increasing trend is more pronounced at far offsets $\left(21^{\circ}-40^{\circ}\right)$ showing high sensitivity. We assumed that the true $V_{\text {shale }}$ and $S_{w}$ are equal to 30 and $20 \%$, respectively. We then try to estimate the true values using the calculated synthetic AVO data, i.e., the reflection coefficient as a function of incidence angle under significant amount of noise/uncertainty conditions. In this particular case, the functional form of the objective function $J(\mathbf{m})$ can be written as:

$$
J(\mathbf{m})=\sum_{i=1}^{40}\left[\frac{R_{i}^{c}(\mathbf{m})-R_{i}^{o}}{\Delta R_{i}}\right]^{2} .
$$

The right hand side of equation (11) is the misfit between the observed and calculated reflection coefficients summed over $0^{\circ}-40^{\circ}$ polar angle of incidence. The source of uncertainty in the seismic data is linked to the acquisition and processing of seismic data. In this study, we have assumed a standard deviation/uncertainty (incorporated through denominator of the right hand side of equation 11) of 10 and $30 \%$ for observed synthetic AVO data and investigated how accurately one can recover the true values of reservoir parameters under Bayesian scheme of inversion discussed in section 3.

During the inversion process, full exploration of posterior PDF has been performed and we have computed all values of $S_{w}$ for each value of $V_{\text {shale }}$ and vice versa. More specifically, we obtained samples of the posterior PDF on the basis of the numerical integration using a constant (non-informative) prior PDF. Figure 8 shows joint PDF for $S_{w}$ and $V_{\text {shale }}$ at $10 \%$ uncertainty in measured AVO data. Figure 9 shows the inversion result in the form of marginal PDFs for reservoir parameters using AVO data with $10 \%$ uncertainty. The peak of marginal distributions for $V_{\text {shale }}$ and $S_{w}$ lies exactly at the true values indicating that our workflow gives us reliable estimates of reservoir parameters in this case. Figure 10 shows joint PDF for $S_{w}$ and $V_{\text {shale }}$ at $30 \%$ uncertainty in measured AVO data. At $30 \%$ standard deviation of synthetic AVO data, the marginal PDF for $S_{w}$ becomes unstable (different local minima for our objective function), i.e., showing more than one peak (figure 11). This character suggests that as the noise in AVO data increases, recovering $S_{w}$ becomes more difficult. The peak for marginal distribution of $V_{\text {shale }}$ lies at the true value suggesting that it can be recoverable even at significant amount of uncertainty in AVO data (figure 11).

A synthetic example related with the simultaneous inversion of correlated Gaussian distribution (Buland and Omre 2003) of $S_{w}$ and $V_{\text {shale }}$ on a map (as in case of real data) having $100 \times 100$ grid blocks is also presented (figure 12). The main aim here is to investigate whether AVO data is able to recover the trend of $S_{w}$ and $V_{\text {shale }}$ at $30 \%$ noise in the data. 


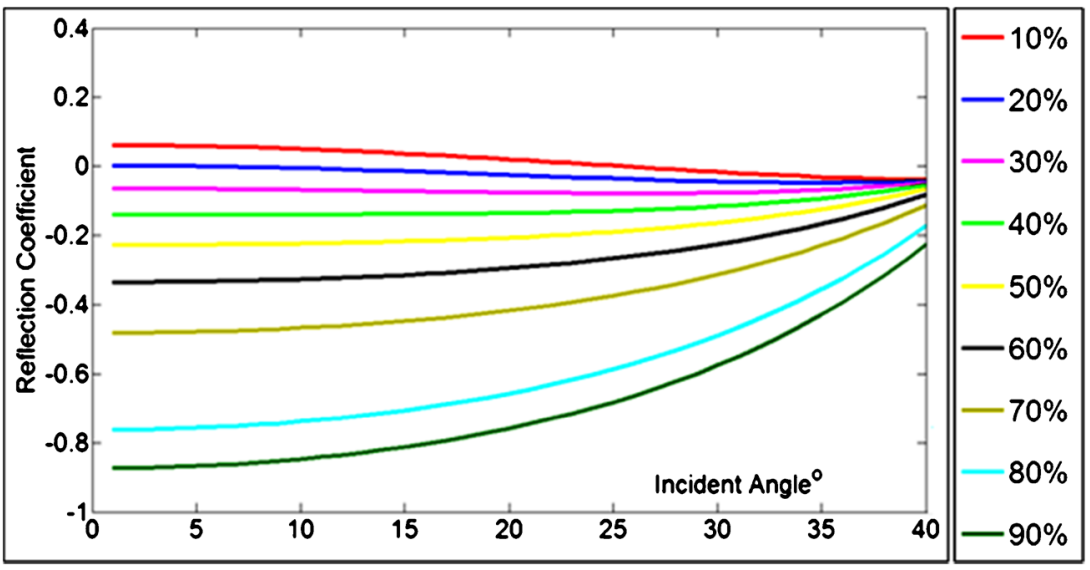

Figure 7. Variation of reflection coefficients as a function of angle for different percentages of $V_{\text {shale }}$ with porosity $=20 \%$, $S_{w}=20 \%$ and overburden properties as given in table 1.

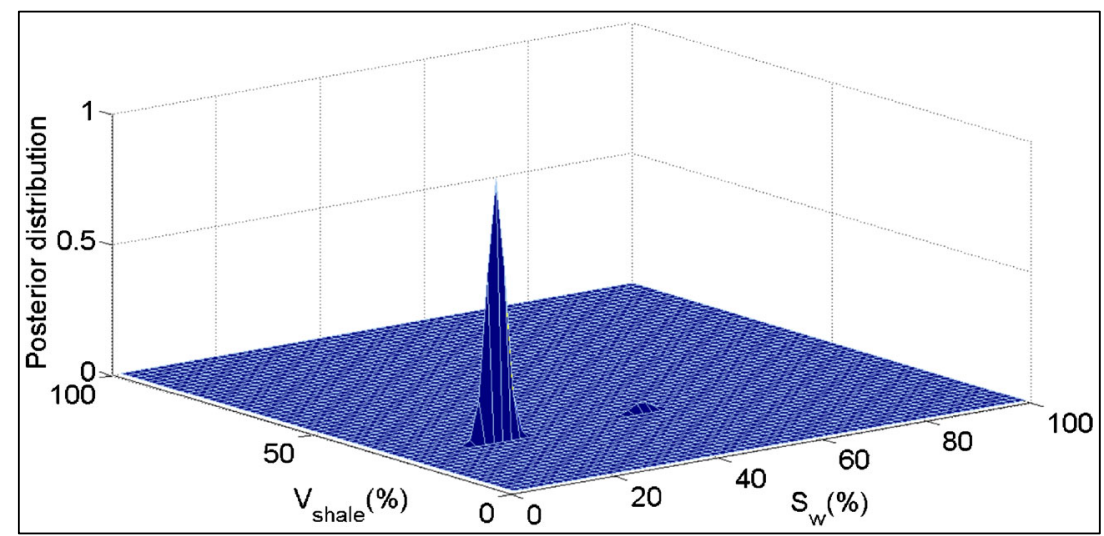

Figure 8. Joint posterior PDF for $S_{w}$ and $V_{\text {shale }}(10 \%$ uncertainty).

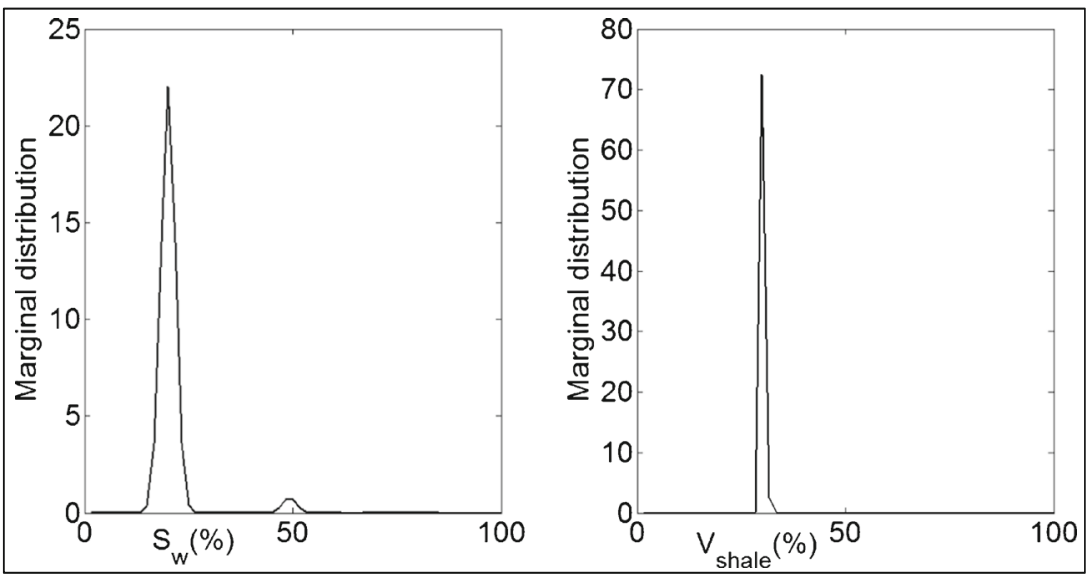

Figure 9. Marginal PDF via numerical integration method for $S_{w}$ and $V_{\text {shale }}(10 \%$ uncertainty).

We have presented both the maximum a posteriori (MAP) solution using systematic search method and the associated uncertainty for each grid block (Tarantola 2005). Our results (figure 12) suggest that it becomes difficult for AVO data to recover the trend for $S_{w}$ at $30 \%$ noise level. For $V_{\text {shale }}$, our workflow is able to recover reliable trend even at this high level of uncertainty. The associated uncertainty in case of $V_{\text {shale }}$ model is less compared to the associated uncertainty of $S_{w}$ model (figure 12), which is in agreement with the inverted results of $S_{w}$ and $V_{\text {shale }}$ in case of a single grid block (figures 8-11). 
The inversion results obtained in the form of marginal PDFs for $V_{\text {shale }}$ and $S_{w}$ have narrow peaks showing the sensitivity of model parameters to uncertainty in the observed data. This can also be attributed to the fact that the change in AVO data (reflection coefficients) is large with change in $V_{\text {shale }}$ as compared to change in $S_{w}$ (figures 6-7). Hence, with the increase of uncertainty, there is a very small change in the marginal distribution for $V_{\text {shale }}$ as compared to the marginal distribution for $S_{w}$ (figures 9 and 11). So, the inversion results presented here should be looked in the context of known (fixed) porosity for background sand along with homogeneous mixing of the fluids (Reuss average). There may be quite different inversion results if one wishes to model the variability in the elastic properties of background sand (e.g., using a range of porosity distribution) along with patchy saturation of the fluid.

It is also important to mention that the testing of workflow presented in this study requires the use of synthetic dataset in the first place. In this research work, we have presented and tested a workflow for estimation of volume of shale and water saturation from synthetic AVO data. It can be interesting to apply this workflow on real data, as it requires sophisticated processing steps on seismic data. In other words, the workflow presented here can be applied to the real data of shaley-sand reservoirs by using pre-stack seismic reflection data gathers at various angles. There are number of factors affecting amplitude of the seismic wave, which require that information about reflection coefficients should be obtained via amplitude preserving migration in anisotropic media (Kiyashchenko et al. 2003; Ursin 2004). The input to rock physics modelling such as mineral/matrix, fluid, porosity and overburden properties can be obtained via the analysis (petrophysical and laboratory) of well log and core data. The next step which can be reported in a subsequent paper could be the direct inversion of seismic waveform data instead of extracting reflection coefficients for water and shale content in conjunction with rock physics modelling.

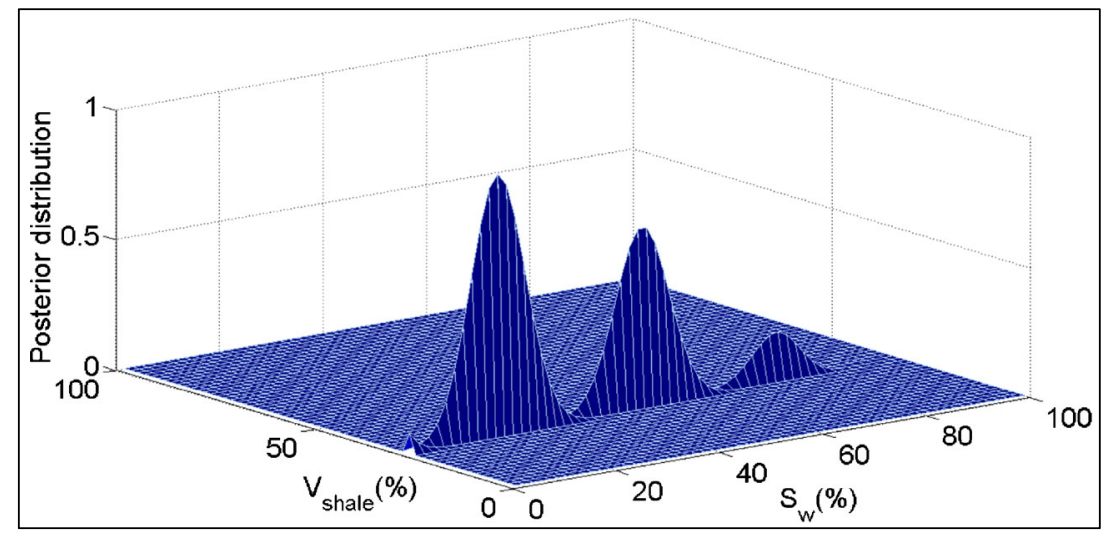

Figure 10. Joint posterior PDF for $S_{w}$ and $V_{\text {shale }}(30 \%$ uncertainty).

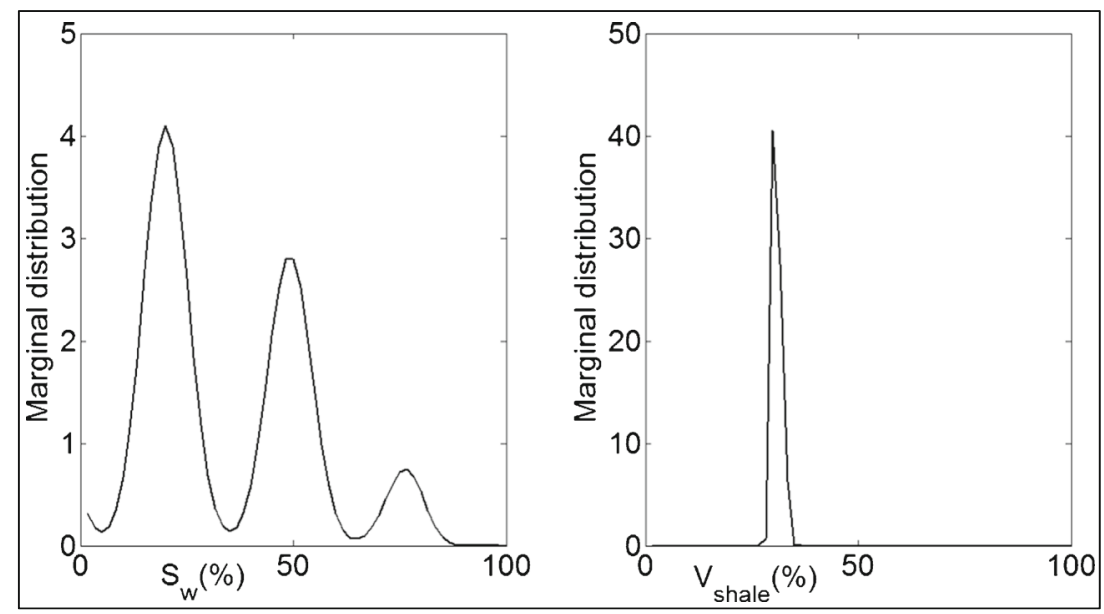

Figure 11. Marginal PDF via numerical integration method for $S_{w}$ and $V_{\text {shale }}$ (30\% uncertainty). 

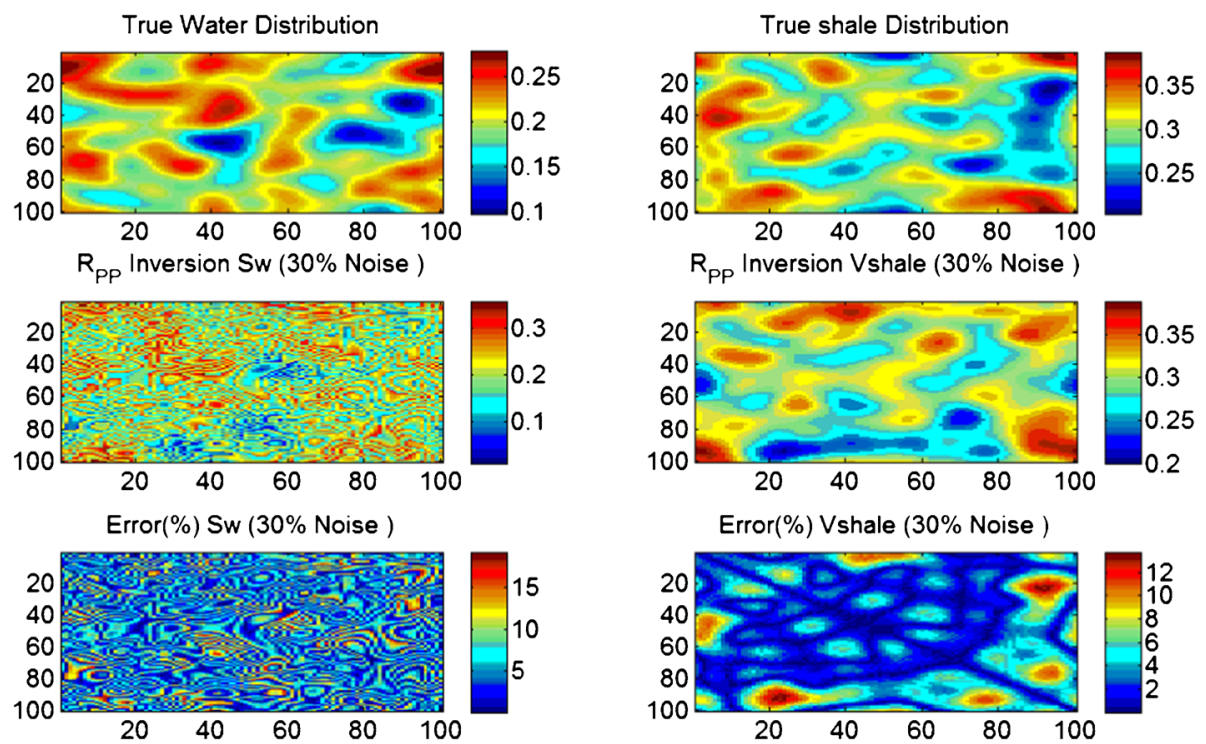

Figure 12. Results of maximum a posteriori distribution for true and inverted reservoir parameters $\left(S_{w}\right.$ and $\left.V_{\text {shale }}\right)$. The upper left and right plots show a Gaussian correlated distribution of reservoir parameters over $100 \times 100$ grid blocks, while middle left and right plots show the inversion results at $30 \%$ uncertainty. The bottom left and right plots show the associated uncertainty of the inversion results. The associated uncertainty in case of water saturation is large due to poor sensitivity of reflection coefficient data with $S_{w}$.

\section{Conclusions}

Reservoir characterization of shaley-sand sequences has always challenged the geoscientists due to their limited seismic resolvability, heterogeneity and anisotropy. Water saturation and volume of shale are among the fundamental reservoir properties of interest for shaley-sand media which relate to the amount of fluid content and accumulating potentials.

Here, we have developed a workflow for reservoir characterization of shaley-sand media by estimating reservoir parameters $\left(V_{\text {shale }}, S_{w}\right)$ from synthetic AVO data provided that sand porosity and overburden properties are known. Furthermore, we have also investigated the level of uncertainty for AVO data to recover these parameters. We have also performed a simultaneous inversion for a correlated Gaussian distribution of $S_{w}$ and $V_{\text {shale }}$ to recover their true trend using maximum a posteriori (MAP) solution on a map having $100 \times 100$ grid blocks.

Our numerical experiments suggest that one can recover $V_{\text {shale }}$ even at higher amount of uncertainty in AVO data, as the peak for marginal distribution lies mostly around the true value. For small amount of uncertainty in AVO data, our workflow gives reliable estimates for $S_{w}$, but as the noise increases in AVO data, it becomes difficult to recover $S_{w}$. For application of this workflow on real data, amplitude preserving migration algorithms should be used to obtain the information about reflection coefficients with angle of incidence.

\section{Acknowledgements}

Hafiz Mubbasher Anwer would like to thank Higher Education Commission (HEC), Pakistan for providing the necessary funding and Department of Earth Sciences, Quaid-i-Azam University, Islamabad, Pakistan for providing the basic requirements to complete this work. Dr Aamir Ali would like to thank Prof. Morten Jakobsen, University of Bergen, Norway for his crucial help during this work. Authors would also like to thank the reviewers for their insightful comments that improved the paper to a great extent.

\section{Appendix A \\ T-matrix approach for composite porous media}

The effective stiffness tensor $\mathbf{C}_{d}^{*}$ of a porous medium with inclusions (shale lenses) for the dry case is given by Jakobsen et al. (2003a):

$$
\mathbf{C}_{d}^{*}=\mathbf{C}^{(0)}+\mathbf{C}_{1}:\left(\mathbf{I}_{4}+\mathbf{C}_{1}^{-1}: \mathbf{C}_{2}\right)^{-1},
$$

where

$$
\mathbf{C}_{\mathbf{1}}=\sum_{r=1}^{N} v^{(r)} \mathbf{t}^{(r)}
$$

and

$$
\mathbf{C}_{\mathbf{2}}=\sum_{r=1}^{N} v^{(r)} \boldsymbol{t}^{(r)}: \mathbf{G}_{\mathbf{d}}: \mathrm{t}^{(r)} v^{(r)} .
$$


In equation (A3) ':' denotes the double scalar product (Auld 1990), $\mathbf{C}^{(\mathbf{0})}$ is the stiffness tensor of the dry porous matrix, $\mathbf{I}_{\mathbf{4}}$ is the (symmetric) identity matrix for second-rank tensors, $v^{(r)}$ is the volume concentration for inclusions of type $r$ (shale lenses) and $\mathbf{G}_{\mathbf{d}}$ tensor is given by the strain Green's function integrated over an ellipsoid determining the symmetry of the correlation function for the spatial distribution of inclusions (Ponte and Willis 1995; Jakobsen et al. 2003a). The T-matrix for a single inclusion (shale lens) of type $r$ is given by Jakobsen et al. (2003a):

$$
\mathrm{t}^{(\mathrm{r})}=\left(\mathbf{C}^{(r)}-\mathbf{C}^{(\mathbf{0})}\right):\left[I_{4}-\mathbf{G}^{(r)}:\left(\mathbf{C}^{(r)}-\mathbf{C}^{(0)}\right)\right]^{-\mathbf{1}} .
$$

Here, $\mathbf{G}^{(\mathbf{r})}$ is a fourth-rank tensor given by the strain Green's function for a material with properties given by $\mathbf{C}^{(\mathbf{0})}$ integrated over a characteristic spheroid having the same shape as inclusions of type $r$ (Jakobsen et al. 2003a). $\mathbf{C}^{(r)}$ is the stiffness tensor for the inclusions of type $r$ (stiffness tensors of shale lenses) whose elastic properties for the saturated case were used from Hornby et al. (1994) given in table A1.

\section{Appendix B}

\section{Fluid saturation effects}

In order to calculate the effect of fluid saturation on the effective properties of a shaley-sand medium, we have used the anisotropic relations of Brown and Korringa (1975), which can be written in the symbolic or matrix notation given as:

$$
\mathbf{S}^{*}=\mathbf{S}_{\mathbf{d}}^{*}+\frac{\left(\left(\mathbf{S}_{\mathbf{d}}^{*}-\mathbf{S}_{\mathbf{m}}\right):\left(\mathbf{I}_{2} \otimes \mathbf{I}_{2}\right):\left(\mathbf{S}_{\mathbf{d}}^{*}-\mathbf{S}_{\mathbf{m}}\right)\right)}{\varphi^{\mathbf{o}}\left(\mathbf{I}_{2} \cdot \mathbf{S}_{\mathbf{m}} \cdot \mathbf{I}_{2}-\mathbf{1} / K_{f}\right)-\mathbf{I}_{\mathbf{2}} \cdot\left(\mathbf{S}_{\mathbf{d}}^{*}-\mathbf{S}_{\mathbf{m}}\right) \cdot \mathbf{I}_{\mathbf{2}}} .
$$

Here $\otimes$ denotes the dyadic product, $\mathbf{S}_{\mathbf{m}}$ is the compliance tensor of the solid mineral component (properties of mineral quartz were used in the case of shaley-sand model), $\mathbf{S}_{\mathbf{d}}^{*}$ is the effective compliance tensor for the dry shaley-sand medium and $\mathbf{S}^{*}$ is the effective compliance tensor for the saturated shaley-sand medium. $\varphi^{\circ}$ is the total porosity and $\mathbf{I}_{2}$ is the (symmetric) identity matrix for second-rank

Table A1. Elastic constants of shale used in this study for modelling shale lenses (Jones and Wang 1981; Hornby et al. 1994).

\begin{tabular}{lc}
\hline $\mathrm{C}_{\mathrm{ij}}$ & $\mathrm{GPa}$ \\
\hline $\mathrm{C}_{11}$ & $34.3+1.4$ \\
$\mathrm{C}_{33}$ & $22.7+0.9$ \\
$\mathrm{C}_{44}$ & $5.40+0.8$ \\
$\mathrm{C}_{66}$ & $10.6+1.6$ \\
$\mathrm{C}_{13}$ & $5.30+5.4$ \\
\hline
\end{tabular}

tensors. In the case of a composite porous medium (shaley-sand), which is partially saturated with oil, gas and water, $K_{f}$ may be regarded as the bulk modulus of an effective fluid given by Wood also known as Reuss average (Wood 1955; Mavko et al. 2009):

$$
\frac{1}{K_{f}}=\frac{S_{w}}{K_{w}}+\frac{S_{o}}{K_{o}}+\frac{S_{g}}{K_{g}}
$$

where

$$
S_{w}+S_{o}+S_{g}=1 .
$$

Here $S_{w}, S_{o}$ and $S_{g}$ represent the saturation for water, oil and gas and $K_{w}, K_{o}$ and $K_{g}$ represent the bulk modulus for water, oil and gas. Before proceeding further, it is important to clarify that effects of fluid saturation were introduced in our rock physics modelling prior to the modelling of saturated shale inclusions. This means that the fluid saturation effects are only for the background porous sand body.

\section{References}

Ali A and Jakobsen M 2011a On the accuracy of Rüger's approximation for reflection coefficients in HTI media: Implications for the determination of fracture density and orientation from seismic AVAZ data; J. Geophys. Eng. 8 $372-393$.

Ali A and Jakobsen M 2011b Seismic characterization of reservoirs with multiple fracture sets using velocity and attenuation anisotropy data; J. Appl. Geophys. $\mathbf{7 5}$ 590-602.

Ali A, Shahraini A and Jakobsen M 2011 Improved characterization of fault zones by quantitative integration of seismic and production data; J. Geophys. Eng. 8 259-274.

Ali A, Anwer H M and Hussain M 2015 A comparisonal study in the context of seismic fracture characterization based on effective stiffness and compliance methods; Arab. J. Geosci. 8 4117-4125.

Alimoradi A, Moradzadeh A and Bakhtiari M R 2011 Methods of water saturation estimation: Historical perspective; J. Petrol. Gas Eng. 2(3) 45-53, doi: 10.1007/ s12517-014-1478-8.

Alves T M, Kurtev K, Moore G F and Strasser M 2014 Assessing the internal character, reservoir potential and seal competence of mass-transport deposits using seismic texture: A geophysical and petrophysical approach; $A m$. Assoc. Petrol. Geol. Bull. 98 793-824.

Asim S, Ahmad S, Zhu P, Naseer M T and Butt M 2016 Spectral decomposition application for analyzing the structure and the reservoir potential: A case study of Penobscot, Nova Scotia offshore, Canada; Arab. J. Geosci. 9 1-20.

Aster R C, Borchers B and Thurber C H 2005 Parameter estimation and inverse problems; Elsevier Academic Press.

Auchter N C, Romans B W and Hubbard S M 2016 Influence of deposit architecture on intrastratal deformation, slope deposits of the Tres Pasos Formation, Chile; Sedim. Geol. 341 13-26.

Auld B A 1990 Acoustic Fields and Waves in Solids; Krieger Publishing. 
Avseth P, Mukerji T and Mavko G 2005 Quantitative seismic interpretation: Applying rock physics tools to reduce interpretation risk; Cambridge University Press.

Bachrach R 2006 Joint estimation of porosity and saturation using stochastic rock-physics modelling; Geophys. 71(4) 53-63.

Besheli S A, Urosevic M and Li R 2005 The effect of seismic anisotropy on reservoir characterization; In: $2005 S E G$ Annual Meeting, Society of Exploration Geophysicists.

Bjørlykke K 2015 Unconventional hydrocarbons: Oil shales, heavy oil, tar sands, shale oil, shale gas and gas hydrates; In: Petroleum Geoscience, pp. 581-590.

Brown R J S and Korringa J 1975 On the dependence of elastic properties of a porous rock on the compressibility of the pore fluid; Geophys. 40 608-616.

Buland A and Omre H 2003 Bayesian linearized AVO inversion; Geophys. 68 185-198.

Cardona R 2002 Two theories for fluid substitution in porous rocks with aligned cracks; In: 2002 SEG Annual Meeting, Society of Exploration Geophysicists.

Clavier C, Coates G and Dumanoir J 1984 Theoretical and experimental basis for the dual-water model for interpretation of shaly-sands; Soc. Petrol. Eng. J. 24(1) 153-168.

Dandekar A Y 2013 Petroleum reservoir rock and fluid properties; CRC press.

Downton J, Gray D and Zuk T 2007 Visualizing AVAZ parameter estimates and uncertainty; CSPG CSEG Convention, pp. 256-259.

Diasty W S, El Beialy S Y, Mostafa A R, Ghonaim A A and Peters K E 2015 Crude oil geochemistry and source rock potential of the upper Cretaceous-Eocene succession in the Belayim oilfields, Central Gulf of Suez, Egypt; J. Petrol. Geol. 38 193-215.

Feng H and Bancroft J C 2006 AVO principles, processing and inversion; CREWES Research Report 18 1-19.

Haldorsen H H, Chang D M and Begg S H 1987 Discontinuous vertical permeability barriers: A challenge to engineers and geologists; In: North sea oil and gas reservoirs, pp. 127-151.

Herron M M 1986 Mineralogy from geochemical well logging; Clays Clay. Miner. 34(2) 204-213.

Hornby B E, Larry M, Schwartz and Hudson J A 1994 Anisotropic effective-medium modelling of the elastic properties of shales; Geophys. 59(10) 1570-1583.

Ikelle L T, Yung S K and Daube F 1993 2-D random media with ellipsoidal autocorrelation functions; Geophys. 58 1359-1372.

Jakobsen M and Johansen T A 1999 A test of ANNIE based on ultrasonic measurements on a shale; J. Seismol. Explor. 8 77-89.

Jakobsen M and Johansen T A 2000 Anisotropic approximations for mud rocks: A seismic laboratory study; Geophys. 65 1711-1725.

Jakobsen M, Hudson J A and Johansen T A 2003a T-matrix approach to shale acoustics; Geophys. J. Int. 154 533-558.

Jakobsen M, Johansen T A and McCann C 2003b The acoustic signature of fluid flow in complex porous media; J. Appl. Geophys. 54 219-246.

Javanshir R J, Riley G W, Duppenbecker S J and Abdullayev N 2015 Validation of lateral fluid flow in an over pressured sand-shale sequence during development of Azeri-Chirag-Gunashli oil field and Shah Deniz gas field: South Caspian Basin, Azerbaijan; Mar. Petrol. Geol. 59 593-610.

Jones L E A and Wang H F 1981 Ultrasonic velocities in Cretaceous shales from the Williston Basin; Geophys. 46 288-297.

Kiyashchenko D A, Kashtan B and Plessix R E 2003 The amplitude preserving migration in anisotropic media and sensitivity analysis; In: 65th EAGE Conference 83
Exhibition, European Association of Geoscientists and Engineers, 43p.

Kneller B, Dykstra M, Fairweather L and Milana J P 2016 Mass-transport and slope accommodation: Implications for turbidite sandstone reservoirs; Am. Assoc. Petrol. Geol. Bull. 100 213-235.

Kumar D and Hoversten G M 2012 Geophysical model response in shale gas; Geohorizons 17(1) 31-37.

Lim J S 2005 Reservoir properties determination using fuzzy logic and neural networks from well data in offshore Korea; J. Petrol. Sci. Eng. 49 182-192.

Luo X, Lin Y, Wu L and Yao F 2005 Estimation of anisotropy parameters in VTI media from surface Pwave seismic data and VSP in Tarim Basin, China; In: 2005 SEG Annual Meeting, Society of Exploration Geophysicists.

Mavko G, Mukerji T and Dvorkin J 2009 The rock physics handbook: Tools for seismic analysis in porous media; Cambridge University Press.

Patnode H W and Wyllie M R J 1950 The presence of conductive solids in reservoir rock as a factor in electric log interpretation; J. Petrol. Technol. 2(2) 47-52.

Ponte C P and Willis J R 1995 The effect of spatial distribution on the effective behaviour of composite materials and cracked media; J. Mech. Phys. Solids 43 1991-2051.

Potter P E, Maynard J and Pryor W A 2012 Sedimentology of shale: Study guide and reference source; Springer Science \& Business Media.

Poupon A and Leveaux J 1971 Evaluation of water saturations in shaly formations; In: SPWLA 12th Annual Logging Symposium, Society of Petrophysicists and Well-Log Analysts.

Quirein J, Donderici B, Torres D, Murphy E and Witkowsky J 2012 Evaluation of general resistivity density-based saturation in thin, laminated sand-shale sequences; In: AAPG Int. Conf. Exhib. "Asia Pacific Resources: Fueling the Future", Singapore.

Rüger A 1998 Variation of P-wave reflectivity with offset and azimuth in anisotropic media; Geophys. 63 935-947.

Rüger A 2002 Reflection coefficients and azimuthal AVO analysis in anisotropic media; Geophysical Monograph Series.

Sams M S and Andrea M 2001 The effect of clay distribution on the elastic properties of sandstones; Geophys. Prospect. 49 128-150.

Sayers C M 1998 Long-wave seismic anisotropy of heterogeneous reservoirs; Geophys. J. Int. 132 667-673.

Schwartz L M and Sen N 1988 Electrical conduction in partially saturated shaly formations; In: 63rd Annual Conference of SPE, Society of Petroleum Engineers.

Sen P N, Goode P A and Sibbit A M 1988 Electrical conduction in clay bearing sandstones at low and high salinities; J. Appl. Phys. 63 4832-4840.

Sengupta M and Bachrach R 2007 Uncertainty in seismicbased pay volume estimation: Analysis using rock physics and Bayesian statistics; The Leading Edge 26(1) 184-189.

Soto B R, Arteaga D, Martin C and Rodriguez F 2010 The correct shale-volume characterization increases hydrocarbon reserves: Case study of cretaceous formation, Lake Maracaibo, Venezuela; In: SPE Latin American and Caribbean Petroleum Engineering Conference, Society of Petroleum Engineers.

Stovas A, Landrø M and Avseth P 2004 Estimation of netto-gross and fluid saturation in a fine-layered sand-shale sequence tested on offshore Brazil data; In: 2004 SEG Annual Meeting, Society of Exploration Geophysicists.

Takahashi I 2000 Quantifying information and uncertainty of rock property estimation from seismic data; $\mathrm{PhD}$ thesis, Stanford University, California, USA. 
Tarantola A 2005 Inverse problem theory and methods for model parameter estimation; Society for Industrial and Applied Mathematics.

Thomsen L 1995 Elasticity anisotropy due to aligned cracks in porous rock; Geophys. Prospect. 43 805-829.

Tsvankin I 1997a Anisotropic parameters and P-wave velocity for orthorhombic media; Geophys. 62 1292-1309.

Tsvankin I 1997b Reflection move-out and parameter estimation for horizontal transverse isotropy; Geophys. 62 614-629.

Uden R, Dvorkin J, Walls J and Carr M 2004 Lithology substitution in a sand/shale sequence; In: 17th Geophysical Conference and Exhibition, Australian Society of Exploration Geophysics.

Ursin B 2004 Parameter inversion and angle migration in anisotropic elastic media; Geophys. 69 1125-1142.

Van Golf-Racht T D 1982 Fundamentals of fractured Reservoir Engineering; Elsevier.

Vernik L, Fisher D and Bahret S 2002 Estimation of net-togross from $\mathrm{P}$ and $\mathrm{S}$ impedance in deep water turbidites; The Leading Edge 21 380-387.
Wang L, Dai H, Li X Y, Sun X and Daqing P 2006 Estimating anisotropic parameters from PS converted-wave data: A case study; SEG Technical Program Expanded Abstracts $251218-1222$.

Wang Y 2011 Seismic anisotropy estimated from P-wave arrival times in crosshole measurements; Geophys. J. Int. 184 1311-1316.

Waxman M H and Smits L J M 1968 Electrical conductivities in oil-bearing shaly sands; Soc. Petrol. Eng. J. 8(2) $107-122$.

Winsauer W O and McCardell W M 1953 Ionic doublelayer conductivity in reservoir rock; J. Pet. Technol. 5(5) $129-134$.

Wood A W 1955 A textbook of sound; McMillan Co., New York.

Worthington P F 1985 The evolution of shaley-sand concepts in reservoir evaluation; The Log Analyst SPWLA 26 $23-40$.

Wyllie M R J and Southwick P F 1954 An experimental investigation of the SP and resistivity phenomena in dirty sands; J. Petrol. Technol. 6(1) 44-57.

MS received 14 July 2016; revised 16 December 2016; accepted 20 December 2016

Corresponding editor: PAWAN DeWANGan 